\title{
GTPases : visualisation spatio-temporelle des réponses de Ras et Rapl
}

Un des rêves du biologiste, visualiser dans le temps et l'espace à l'intérieur d'une cellule les réponses à une stimulation extracellulaire, est-il en passe de devenir réalité? Dans un article récemment publié dans la revue $N$ ature [1], I'équipe de Matsuda nous permet de faire un pas de plus pour nous rapprocher de la compréhension de ces phénomènes extrêmement complexes.

Les cellules répondent à de nombreux signaux extracellulaires (hormones, facteurs de croissance, etc.) par la production de seconds messagers intracellulaires tels l'élévation de la concentration intracellulaire en AMPc ou en ions $\mathrm{Ca}^{2+}$ libres, ou l'activation des GTPases de la famille Ras, protéines qui agissent comme des interrupteurs moléculaires (voir figure 1). Dans tous les cas, il en résulte I'activation de voies effectrices en aval qui sont responsables de la mise en œuvre des réponses biologiques à ces signaux. Depuis de nombreuses années, on savait suivre biochimiquement la production de ces seconds messagers, ou encore, plus récemment, la proportion des GTPases de la famille Ras qui, sous l'impulsion du signal, adoptent une conformation active liée au GTP. Toutefois, ces méthodes biochimiques ne permettent qu'une mesure globale de la cinétique de réponse à la stimulation au sein de la population cellulaire.

Afin de visualiser à l'intérieur d'une cellule le déroulement des réponses dans l'espace et dans le temps, on a produit et introduit dans les cellules vivantes des indicateurs fluorescents, détectables au microscope, dont le niveau de fluorescence varie avec

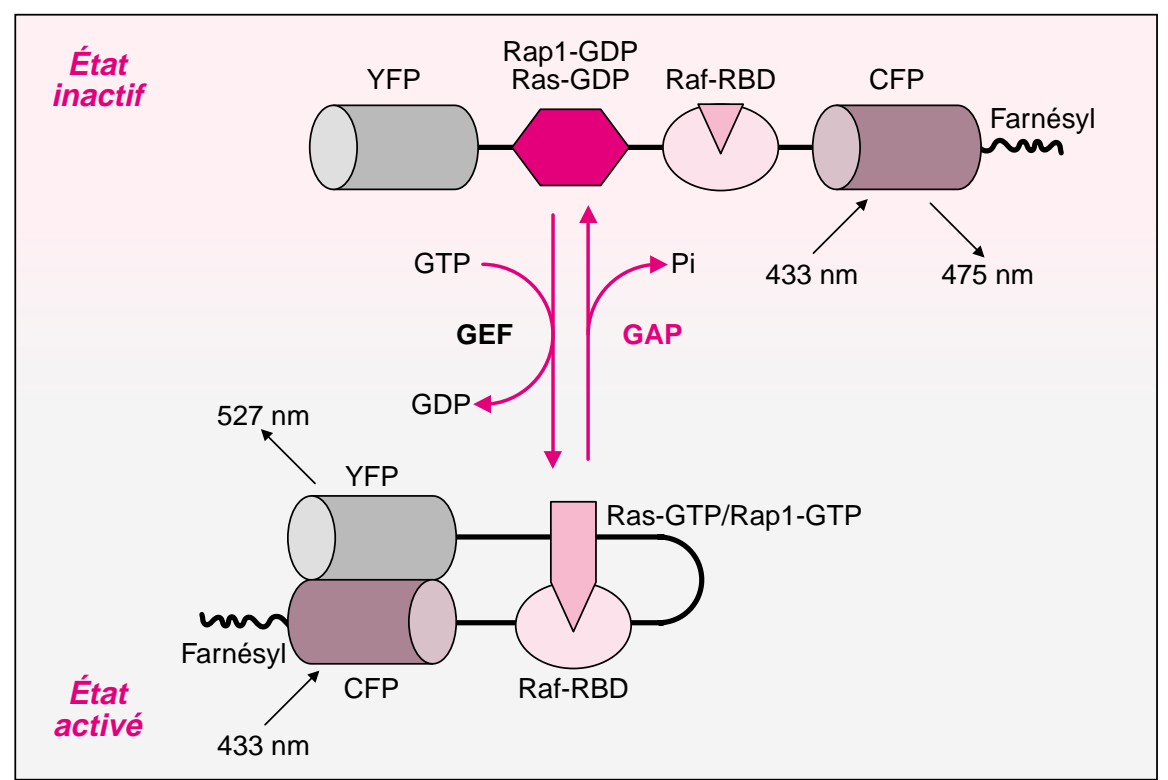

Figure 1. Représentation de la molécule hybride concue par Mochizuki et al. pour visualiser dans les cellules par transfert de fluorescence l'activation des GTPases Ras et Rap1. En haut, la molécule dans sa conformation inactive, en bas dans sa conformation active, permettant le transfert de fluorescence. YFP: yellow fluorescent protein; CFP: cyan fluorescent protein; les Iongueurs d'onde d'excitation et d'émission sont indiquées ( $\mathrm{nm}$ ).

rer. La technique de choix, développée en particulier par l'équipe de Roger Tsien pour suivre la production d'AMPc $[2,3]$ et d'ions $\mathrm{Ca}^{2+}$ libres $[4,5]$, a consisté à utiliser le transfert de fluorescence (FRET ou fluorescence resonnance energy transfer) entre deux molécules proches portant des fluorophores différents et compatibles [6]. Lorsque le fluorophore de plus basse longueur d'onde est excité, il émet des photons qui peuvent être captés par un second fluorophore de longueur d'onde d'excitation correspondante, qui émettra à son tour un signal fluorescent de longueur d'onde supérieure.
Un tel transfert de fluorescence $n$ 'est possible que si les deux partenaires sont en contact étroit, et permet donc de mesurer leur interaction par le rapport de la fluorescence de transfert (FRET émis par le fluorophore de longueur d'onde d'émission plus élevée) à la fluorescence d'émission du fluorophore de longueur d'onde la plus faible. L'utilisation de la GFP (green fluorescent protein), protéine spontanément fluorescente de la méduse Aequorea victoria, et de ses dérivés de couleurs variées (cyan, jaune et bleu) a permis de raffiner cette technique en construisant de tels indicateurs de 
fluorescence codés génétiquement et adressables à des localisations intracellulaires spécifiques. Ces indicateurs résultent de la fusion entre une protéine fluorescente bleue (BFP) ou cyan (CFP) avec un premier partenaire, ainsi que de la fusion entre le second partenaire et une protéine fluorescente verte (GFP) ou jaune (YFP) ; la co-expression, par transfection transitoire, des deux molécules chimériques au sein d'une même cellule permet le transfert de la fluorescence de la BFP ou de la CFP vers la FGP ou la YFP Iorsqu'elles sont en contact.

De nombreux signaux extracellulaires convergent pour activer les GTPases de la famille Ras via plusieurs protéines régulatrices. Toutefois, si tant de signaux différents activent ces GTPases, on ne sait pas comment elles aboutissent à la création de réponses spécifiques à chacun de ces signaux. Afin de suivre dans le temps et l'espace l'activation de deux GTPases proches, Ras et Rap1, en réponse à la stimulation des cellules par un mitogène, Mochizuki et al. ont perfectionné cette technique de transfert de fluorescence. Ils ont exprimé dans des cellules une molécule hybride comprenant la GTPase Ras ou Rap1, le domaine de liaison à Ras et Rap de la protéine Raf-1 (Raf-RBD) ainsi que deux protéines fluorescentes, la CFP et la YFP (figure 1). L'activation de la GTPase, qui passe de l'état lié au GDP à l'état lié au GTP, lui permet alors d'interagir avec son effecteur le Raf-RBD, ce qui a pour effet de rapprocher les deux fluorophores provoquant ainsi le transfert de fluorescence de la CFP vers la YFP. Les auteurs ont enregistré ces images sur des cellules vivantes; la mesure normalisée des intensités de FRET permet de visualiser à l'intérieur de chaque cellule la progression de l'activation de ces GTPases en réponse à une stimulation.

Comme on pouvait s'y attendre, la GTPase Ras est activée à la membrane plasmique quelques minutes après la stimulation de cellules fibroblastiques COS par I'EGF (epidermal growth factor). La protéine activée se retrouve ensuite à travers l'ensemble du cytoplasme, reflétant probablement $\mathrm{m} / \mathrm{s} \mathrm{n}^{\circ} 12$, vol. 17, décembre 2001

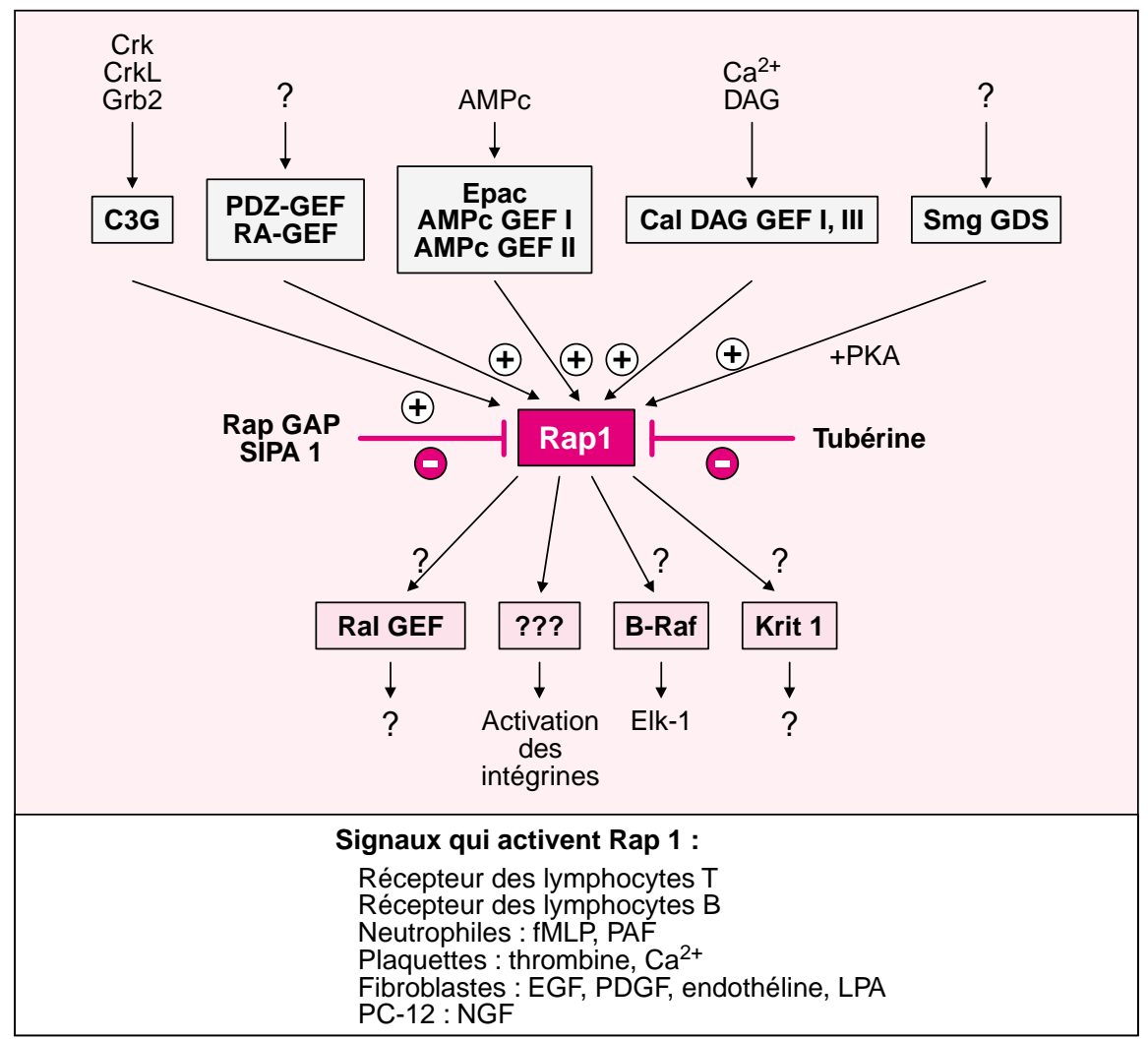

Figure 2. La voie de transduction Rapl. Rap1 est activée par de nombreux GEF en réponse à une grande diversité de signaux. Bien que plusieurs de ses protéines effectrices soient connues, les effets physiologiques de l'activation de Rapl ne sont pas encore bien compris.

I'internalisation des complexes de signalisation actifs. Dans les cellules neuroendocrines différenciées PC-12, I'activation de Ras par un agent différenciant, le NGF (nerve growth factor), suit une cinétique comparable. Toutefois, plusieurs heures après la stimulation, on retrouve la protéine Ras activée à l'extrémité des neurites. Des expériences de photo-décoloration montrent qu'il ne s'agit pas de la rétention de protéines Ras actives, mais vraisemblablement de l'activation continuelle de molécules Ras qui oscillent entre conformation inactive et active. En revanche, la protéine Rap1, qui avait été localisée dans des compartiments intracellulaires $[7,8]$, est activée avec une cinétique comparable à Ras dans la région périnucléaire des cellules. Contrairement au cas de Ras, il semble que l'activation de Rapl nécessite la mise en jeu des étapes précoces de l'endocytose [ 1 ,
9], ce qui pourrait permettre aux facteurs d'échange activés d'atteindre leur cible intracellulaire. L'activation intracellulaire de Rap1 pourrait également résulter de l'action de seconds messagers diffusibles tels les ions $\mathrm{Ca}^{2+}$ libres [10]. Cette étude nous apprend donc que, en réponse à un stimulus prolifératif, deux GTPases proches, Ras et Rap1, sont activées dans des régions bien délimitées de la cellule et exclusives l'une de l'autre. De plus, leurs cinétiques d'activation peuvent être différentes, notamment à l'extrémité des neurites des cellules PC-12 stimulées par le NGF.

Les études à présent classiques d'interaction protéine-protéine permettent de déchiffrer les circuits des voies de transduction, en établissant le cheminement, parfois complexe, des signaux de molécule en molécule. Les résultats présentés par 
Mochizuki et al. [1] nous éclairent sur la dimension spatio-temporelle de la progression de ces signaux, et suggèrent que cet aspect de la signalisation joue un rôle déterminant sur la multiplicité et la spécificité des réponses cellulaires à un signal donné.

1. Mochizuki N, Yamashita S, Kurokawa K, et al Spatio-temporal images of growth-factor-induced activation of Ras and Rap1. Nature 2001; 411 1065-8.

2. Adams SR, H arootunian AT, Buechler YJ, Taylor S.S, Tsien RY. Fluorescence ratio imaging of cyclic AMP in single cells. Nature 1991; 349: 694-7.
3. Zaccolo M, De Giorgi $F$, Cho $\mathrm{CY}$, et al. A genetically encoded, fluorescent indicator for cyclic AMP in living cells. Nat Cell Biol 2000; $2: 25-9$.

4. Miyawaki A, Griesbeck 0 , Heim R, Tsien RY Dynamic and quantitative $\mathrm{Ca} 2+$ measurements using improved cameleons. Proc Natl Acad Sci USA 1999: 96 : 2135-40.

5. Miyawaki A, Llopis J, Heim R, et al. Fluorescent indicators for $\mathrm{Ca}^{2+}$ based on green fluorescent proteins and calmodulin. N ature1997; 388: 882-7.

6. Miyawaki A, Tsien RY. Monitoring protein conformations and interactions by fluorescence resonance energy transfer between mutants of green fluorescent protein. M ethods Enzymol 2000; 327: 472-500

7. Béranger F, Goud B, Tavitian A, de Gunzburg J. Association of the ras-antagonistic Rap1/ Krev-1 proteins with the Golgi complex. Proc Natl Acad Sci USA $1991 ; 88$ : 1606-10.

8. Pizon V, Desjardins, M, Bucci C, Parton, R.G, Zerial M. Association of Rapla and Raplb pro- teins with late endocytic/ phagocytic compartments and Rap2a with the Golgi complex. J Cell Sci 1994; 107: 1661-70.

9. York RD, Yao H, Dillon $\mathrm{T}$, et al. Rap1 mediates sustained MAP kinase activation induced by nerve growth factor. Nature 1998; 392: 622-6.

10. Bos, J.L. All in the family ? New insights and questions regarding interconnectivity of Ras, Rap1 and Ral. Embo J 1998; 17: 6776-82.

\section{Jean de G unzburg}

Inserm U. 528, Institut Curie, Section recherche, 26, rue d'UIm, 75248 Paris Cedex 5, France.
Les Druzes d'Israël et la P. cadhérine. Une des nombreuses formes d'hypotrichose observées chez l'homme est associée à une dystrophie maculaire qui évolue vers une cécité précoce. II s'agit de I'HJMD (pour hypotrichosis with juvenile macular dystrophy). Peu fréquente, son mode de transmission, récessif autosomique, a été démontré par le groupe d'Arnold Munnich qui a pu étudier une fratrie d'origine portugaise dont les parents étaient originaires du même village [1]. Une équipe israélienne vient de découvrir le gène en cause grâce à l'étude de quatre grandes familles druzes vivant au nord d'Israël (cette population musulmane de rite initiatique dérivé de l'I smaïlisme est très fortement endogame) [2]. Après avoir situé le locus en 16q22.1, dans la région où se trouvent rassemblés les gènes codant pour les cadhérines, ces chercheurs ont mis en évidence la même délétion, (981delG), dans le gène $\mathrm{CDH} 3$ chez tous les malades. $\mathrm{Ce}$ gène code pour la $\mathrm{P}$-cadhérine ( $P$ car exprimée dans le placenta) et la mutation doit entraîner la production d'une protéine tronquée, non fonctionnelle. Les cadhérines sont des molécules d'adhérence, constitutives des jonctions adhérentes intercellulaires. Elles comportent cinq domaines extracellulaires, un domaine transmembranaire et une courte terminaison intracellulaire qui interagit avec les filaments d'actine par l'intermédiaire des caténines (m/s 1999, $\left.n^{\circ} 1, p .116-7\right)$. Elles sont impliquées dans le développement des poils et de la rétine, mais seule la P-cadhérine est exprimée dans le follicule pileux, alors que les E- et P-cadhérines sont présentes dans les cellules épithéliales. $\mathrm{Si}$ la E-cadhérine peut partiellement compenser la déficience de la P-cadhérine dans l'épiderme, I'absence de cette dernière dans les follicules pileux aboutit à une alopécie partielle et à des cheveux anormaux de type pseudomoniléthrix (épaisseur irrégulière avec renflements et rétrécissements) et de type pili torti (torsion à $180^{\circ}$ ). Dans la rétine, le rôle de la P-cadhérine n'est pas encore bien connu, mais l'examen du fond d'œil montre des cicatrices atrophiques dans la macula, entourées de zones de dégénérescence pigmentaire. Par quel mécanisme la perte de fonction de la P-cadhérine peut-elle entraîner une atteinte des follicules pileux et de l'épithélium pigmentaire de la rétine? En l'absence de son extrémité intracytoplasmique, perdue dans la protéine tronquée, la fixation avec I'actine du cytosquelette devient impossible et doit inhiber l'action de la $\beta$-caténine qui contrôle la morphogenèse des follicules pileux [3]. Chez la souris adulte, le maintien de l'activité de la $\beta$-caténine (contrôlée par un promoteur de l'épiderme) entraîne la formation de nouveaux follicules (glandes sébacées, papilles dermiques) et les poils continuent à pousser [4]. Toutefois, il ne serait pas judicieux de choisir la souris comme modèle animal de l'HJMD, car la perte de la Pcadhérine n'entraîne chez elle ni hypotrichose, ni dystrophie maculaire [5].

[1. Souied E, et al. Ophtal Genet 1995; 16: 11-5.]

[2. Sprecher $\mathrm{E}$, et al. $\mathrm{N}$ at $\mathrm{G}$ enet 2001; 29: 134-6.]

[3. Huelsken ], et al. Cell 2001; 105: 533-45.]

[4. Gat U, et al. Cell 1998; 95 : 605145.]

[5. Radice GL, et al. ] Cell Biol 1997; 139: 1025-32.] 